\title{
RECONSTRUCTION OF THE ONE-DIMENSIONAL THICK DISTRIBUTION THEORY
}

\author{
YUNYUN YANG
}

\begin{abstract}
The theory of thick distributions (both in dimension 1 and in higher dimensions) was constructed in recent years [7] 27]. However this theory of distributions with one thick point in dimension one is very different from that in higher dimensions. In this paper the author uses the language of asymptotic analysis to reconstruct the 1-dimensional thick distribution theory and to incorporate it into the framework of the higher-dimensional thick distribution theory. Some new concepts and interesting results appear in this paper from viewing singular functions in a different way.
\end{abstract}

\section{INTRODUCTION}

In recent years, some research in mathematics and physics suggests us to consider non-smooth functions as test functions in the theory of distributions. In particular, one considers functions with a point singularity. The emergence of the special point either corresponds to the singularity of the field equations, or to the nonlinearity caused by the multiplication of distributions [1, 2, 3, 4, 12, 13, 27. Classical distribution theory cannot handle these problems.

In the past decades, researchers have done some work to solve these problems. Blanchet and Faye develop such a scheme in the context of finite parts, pseudofunctions and Hadamard regularization, to study the dynamics of point particles in high post-Newtonian approximations of general relativity [2]. Their work was built upon the the discussion by Sellier of Hadamard finite part of smooth functions with one point singularity 22 .

Considering distributions with a point singularity can also be applied to the research of point-source fields. Basic electrodynamics tells us that, because of $\nabla^{2} \frac{1}{r}=-4 \pi \delta(\mathbf{x})$ and the Poisson's equation $\nabla^{2} \Phi(\mathbf{x})=-\rho(\mathbf{x}) / \epsilon_{0}$, we can describe the potential of a point-source field caused by an electron $\rho(\mathbf{x})=q \delta(\mathbf{x})$. Yet applying the Laplace operator $\nabla^{2}=\frac{1}{r^{2}} \frac{\partial}{\partial r} r^{2} \frac{\partial}{\partial r}+\ldots$ directly on $\frac{1}{r}$ cannot give us the desired $\nabla^{2} \frac{1}{r}=-4 \pi \delta(\mathbf{x})$. In order to solve this, Blinder [3] and $\mathrm{Hu}$ [16] suggest to use $\operatorname{sgn}(r) / r$ instead of $1 / r$ to have $\nabla^{2} \frac{\operatorname{sgn}(r)}{r}=-2 \delta(r) / r^{2}=-4 \pi \delta(\mathbf{x})$. Yet $\operatorname{sgn}(r) / r$ and $-2 \delta(r) / r^{2}$ both are NOT well-defined distributions. In fact, they are so called "thick distribution".

Paskusz 20] points out, it is not rigorous to say $H(x) \delta(x)=\frac{1}{2} \delta(x)$ based on $H(x)=H^{2}(x)$. Indeed, taking distributional derivative on both sides of $H(x)=$

Date: August 20, 2019.

Key words and phrases. thick test functions, thick distributions, thick delta functions, Hadmard finite part, asymptotic expansion.

Thanks to Hefei University of Technology to support this research. The grant number from Hefei University of Technology is 407-0371000086. 
$H^{2}(x)$ does give us $2 H(x) \delta(x)=\delta(x)$. Yet, if one continues to multiply both sides with $2 H(x)$ one gets $\delta(x)=2 H(x) \delta(x)=4 H^{2}(x) \delta(x)=4 H(x) \delta(x)$, and then gets $2=4$. In fact, both $H^{2}(x)$ and $H(x) \delta(x)$ are not well-defined distributions. One cannot simply apply distributional derivatives on both sides of the above equation.

Bowen, [4, points out that, when we multiply two distributions, the product rule may not apply. For example, let $n_{j_{k}}=x_{j_{k}} / r$, where $x_{j_{k}}$ is the $j_{k}$-th coordinate and $r$ is the radius $r=\sqrt{x_{1}^{2}+\ldots+x_{n}^{2}}$. We have

$$
\frac{\bar{\partial}}{\partial x_{i}}\left(\frac{n_{j_{1}} n_{j_{2}} n_{j_{3}}}{r^{2}}\right) \neq n_{j_{1}} n_{j_{2}} \frac{\bar{\partial}}{\partial x_{i}}\left(\frac{n_{j_{3}}}{r^{2}}\right)+\frac{n_{j_{3}}}{r^{2}} \frac{\bar{\partial}}{\partial x_{i}}\left(n_{j_{1}} n_{j_{2}}\right) .
$$

where the $\bar{\partial}$ means the distributional derivative. However, the equation holds when one considers "thick distributional derivative".

A one-dimensional thick distribution theory was first developed by Estrada and Fulling in order to solve some of these dilemmas [7]. They define the "thick test function space" as the topological vector space consists of all compactly supported functions which are smooth on $\mathbb{R} \backslash\{a\}$, and whose one-sided derivatives at $x=a$ exist. They defined the "thick distribution space" as the dual space of the thick test function space.

The thick distribution theory of higher dimensions was later developed by Estrada and Yang [27. We also published a series of papers to further develop the application of thick distributions [9, 25, 26, 29. In higher dimensions, we define the "thick test functions" as those compactly supported functions that are smooth on $\mathbb{R}^{n} \backslash\{\mathbf{a}\}$, and which have a strong asymptotic expansion near $\mathbf{a}: \phi(\mathbf{a}+\mathbf{x}) \sim \sum_{j=m}^{\infty} a_{j}(\mathbf{w}) r^{j}$ when $\mathbf{x} \rightarrow \mathbf{0}$, where $a_{j}(\mathbf{w})$ is a smooth function on the unit sphere $\mathbb{S}^{n-1}$. Given a proper topology, the space of all such functions is a topological vector space. We then define the dual space as the "thick distribution space".

One could see that because $\mathbb{R}^{n}(n \geq 2)$ is connected when taking away a single point $\mathbf{a}$, while $\mathbb{R}$ is disconnect doing the same thing, the construction of the higher dimensional thick distribution theory is very different from that of the onedimensional case. One could not talk about "one-sided derivatives" in the higher dimensional case. Instead, we used the asymptotic expansion behavior near the singularity to describe thick test functions.

The purpose of this present article is to try to combine these two seemingly different theories, and to incorporate the one-dimensional thick distribution theory into the framework of the higher dimensional thick distribution theory. This work will give us some insights of the function space itself.

About the notation: $d / d x$ means the ordinary derivative, $\bar{d} / d x$ means the distributional derivative, $d^{*} / d x$ means the thick distributional derivative.

This is the structure of this article: in Section 2 we give a brief review of the established thick distribution theory. Section 3 is the main part of this article, we introduce a new one-dimensional thick distribution theory there, which is more allied with the higher dimensional thick distribution theory, and the established onedimensional thick distribution theory is a special case of the new thick distribution theory. In this section, we firstly identify $\mathbb{R} \backslash\{0\}$ with $\mathbb{S}^{0} \times \mathbb{R}_{+}$, where "S $\mathbb{S}^{0}$ " denotes the "0-dimensional unit sphere", namely, the 2 end-points of the line segment $[-1,1]$. Under this viewpoint, we define the meaning of a function $f(x)=f(\mathbf{w}, r)$ having an asymptotic expansion $\sum_{j=m}^{\infty} a_{j}(\mathbf{w}) r^{j}$ as $r \rightarrow 0^{+}$, where $\mathbf{w} \in \mathbb{S}^{0}$. Then, 
we give a few examples where viewing $f(x)$ as $f(\mathbf{w}, r)$ are quite elegant, as well as a few lemmas discussing a few properties of $f(\mathbf{w}, r)$. In subsection 3.2 we introduce a new thick test function space in one-dimensional case. Under our definition, the established thick test function space in one-dimensional case is a closed subspace. In subsections 3.3 and 3.4, we reconstruct the one-dimensional thick distribution theory, and provide a few examples of the new thick distributions. At the end of the article we provide a solution of a problem pointed out by Paskusz, of multiplying Schwartz distributions, in the context of the new one-dimensional thick distributoin theory.

\section{REview of the ESTABlished 1-Dim AND HIGHER DIMENSIONAL THICK}

\section{DISTRIBUTION THEORY}

2.1. One-dimensional thick distribution theory. One-dimensional thick distribution theory was developed by Estrada and Fulling in 2007 [7]. In their paper, the "thick test function space" is defined as the topological vector space consists of all compactly supported functions which are smooth on $\mathbb{R} \backslash\{a\}$, and whose onesided derivatives at $x=a$ exist. One denotes thick test function space $\mathcal{D}_{*, a}(\mathbb{R})$. Given a proper topology, the usual test function space $\mathcal{D}(\mathbb{R})$ is a closed subspace of $\mathcal{D}_{*, a}(\mathbb{R})$. Then they define the dual space of $\mathcal{D}_{*, a}(\mathbb{R})$ as "thick distribution space", denoted $\mathcal{D}_{*, a}^{\prime}(\mathbb{R})$. If $a=0$, we simply denote the space of thick test functions and thick distributions $\mathcal{D}_{*}(\mathbb{R})$ and $\mathcal{D}_{*}^{\prime}(\mathbb{R})$, respectively.

By the Hahn-Banach theorem, the usual distribution space $\mathcal{D}^{\prime}(\mathbb{R})$ is just a projection of $\mathcal{D}_{*, a}^{\prime}(\mathbb{R})$. Namely, any usual distribution $f$ has a lifting $\widetilde{f}$ in $\mathcal{D}_{*, a}^{\prime}(\mathbb{R})$, such that $\pi(\widetilde{f})=f$, where $\pi$ is the projection operator.

An example of thick distribution in $\mathcal{D}_{*}^{\prime}(\mathbb{R})$ would be $\delta_{+}(x)$ : for any thick test function $\phi(x)$, we have

$$
\left\langle\delta_{+}(x), \phi(x)\right\rangle=\phi_{+}(0),
$$

where $\phi_{+}(0)$ denotes $\lim _{x \rightarrow 0^{+}} \phi(x)$, the rightside limit of $\phi(x)$; and $\phi_{-}(0)$ denotes $\lim _{x \rightarrow 0^{-}} \phi(x)$. Moreover, one can define an extension of the Dirac delta function as:

$$
\delta_{*, \lambda}(x)=\lambda \delta_{+}(x)+(1-\lambda) \delta_{-}(x) .
$$

Namely, the projection of $\delta_{*, \lambda}(x)$ onto the usual distribution space is the Dirac delta function $\delta(x)$ : if we use $i: \mathcal{D}(\mathbb{R}) \hookrightarrow \mathcal{D}_{*}(\mathbb{R})$ to denote the inclusion and $\pi$ : $\mathcal{D}_{*}^{\prime}(\mathbb{R}) \rightarrow \mathcal{D}^{\prime}(\mathbb{R})$ the projection, then one clearly sees that, for any $\phi(x) \in \mathcal{D}(\mathbb{R})$, we have $\phi_{+}(0)=\phi_{-}(0)=\phi(0)$, then,

$$
\begin{aligned}
\left\langle\pi\left(\delta_{*, \lambda}(x)\right), \phi(x)\right\rangle & =\left\langle\delta_{*, \lambda}(x), i(\phi(x))\right\rangle \\
& =\lambda \phi_{+}(0)+(1-\lambda) \phi_{-}(0) \\
& =\phi(0)=\langle\delta(x), \phi(x)\rangle .
\end{aligned}
$$

In particular, if $\lambda=\frac{1}{2}$,

$$
\delta_{*, 1 / 2}(x)=\frac{1}{2} \delta_{+}(x)+\frac{1}{2} \delta_{-}(x) .
$$

Let $\mathcal{S}_{*}(\mathbb{R})$ denote the space of smooth functions with a thick point at the origin, and with fast decay at infinity. Then in [7] Estrada and Fulling proved that the space of Fourier transform of $\mathcal{S}_{*}(\mathbb{R})$ would be the space $\mathcal{W}(\mathbb{R})$. Namely, the space 
consists of those functions $\psi \in C^{\infty}(\mathbb{R})$ that admits an asymptotic expansion of the type

$$
\psi(x) \sim \sum_{n=1}^{\infty} c_{n} x^{-n}, \quad \text { as }|x| \rightarrow \infty,
$$

for some constants $c_{1}, c_{2}, c_{3}, \ldots$. The correponding dual space $\mathcal{W}^{\prime}$ is therefore the Fourier transform of the tempered thick distributions $\mathcal{S}_{*}^{\prime}$.

2.2. Higher dimensional thick distribution theory. In paper [27, Yang and Estrada developed a theory of thick distributions in dimension $n \geq 2$. There is a essential difference between the topology of $\mathbb{R}$ and that of $\mathbb{R}^{n}$ : taking away a point in $\mathbb{R}$ will make it into two disconnected parts while doing the same thing for $\mathbb{R}^{n}$ will not. Hence we cannot use the so called "jump discontinuity" to describle singularities of functions in $\mathbb{R}^{n}$. Therefore, the theory of thick distributions in higher dimensions is quite different from the established one-dimensional theory.

Definition 1. Let $\phi$ be defined in $\mathbb{R}^{n} \backslash\{\mathbf{0}\}$. We say that $\phi$ has the asymptotic expansion $\sum_{j=m}^{\infty} a_{j}(\mathbf{w}) r^{j}$ as $\mathbf{x} \rightarrow \mathbf{0}$ if for all $M \geq m, M \in \mathbb{Z}$,

$$
\lim _{r \rightarrow 0^{+}}\left|\phi(\mathbf{x})-\sum_{j=m}^{M} a_{j}(\mathbf{w}) r^{j}\right| r^{-M}=0, \quad \text { uniformly on } \mathbf{w} \in \mathbb{S} \text {. }
$$

In this case we write $\phi(\mathbf{x}) \sim \sum_{j=m}^{\infty} a_{j}(\mathbf{w}) r^{j}$ as $\mathbf{x} \rightarrow \mathbf{0}$.

Let $\mathbf{p} \in \mathbb{N}^{n}$ denote a multi-index and $(\partial / \partial \mathbf{x})^{\mathbf{p}}=\left(\partial^{|\mathbf{p}|}\right) / \partial x_{1}^{p_{1}} \ldots \partial x_{n}^{p_{n}},|\mathbf{p}|=p_{1}+$ $\ldots+p_{n}$. If $a_{j}(\mathbf{w}) \in C^{\infty}\left(\mathbb{S}^{n-1}\right)$ is a smooth function on the unit sphere $\mathbb{S}^{n-1}$, then applying $(\partial / \partial \mathbf{x})^{\mathbf{p}}$ on $a_{j}(\mathbf{w}) r^{j}$ we will obtain another homogeneous function of degree $j-|\mathbf{p}|$, which we denote $a_{j-|\mathbf{p}|, \mathbf{p}}(\mathbf{w}) r^{j-|\mathbf{p}|}$. Hence formally applying $(\partial / \partial \mathbf{x})^{\mathbf{p}}$ on $\sum_{j=m}^{\infty} a_{j}(\mathbf{w}) r^{j}$ will give us another expansion of the form $\sum_{j=m-|\mathbf{p}|}^{\infty} a_{j, \mathbf{p}}(\mathbf{w}) r^{j}$. In general, asymptotic expansions cannot be differentiated [8]; if the asymptotic expansion of the differentiation of the function is the same as the term-by-term differentiation of the expansion, then the expansion is called "strong".

Definition 2. Let $\phi \in C^{\infty}\left(\mathbb{R}^{n} \backslash\{\mathbf{0}\}\right)$. We say that the expansion $\phi(\mathbf{x}) \sim \sum_{j=m}^{\infty} a_{j}(\mathbf{w}) r^{j}$ as $\mathbf{x} \rightarrow 0$ is strong if for each $\mathbf{p} \in \mathbb{N}^{n}$ the asymptotic development of $(\partial / \partial \mathbf{x})^{\mathbf{p}} \phi(\mathbf{x})$ as $\mathbf{x} \rightarrow \mathbf{0}$ exists and equals $\sum_{j=m-|\mathbf{p}|}^{\infty} a_{j . \mathbf{p}}(\mathbf{w}) r^{j}$, the term-by-term differentiation of $\sum_{j=m}^{\infty} a_{j}(\mathbf{w}) r^{j}$.

Definition 3. Let $\mathcal{D}_{*, \mathbf{a}}\left(\mathbb{R}^{n}\right)$ denote the vector space of all smooth functions $\phi$ defined in $\mathbb{R}^{n} \backslash\{\mathbf{a}\}$, with support of the form $K \backslash\{\mathbf{a}\}$, where $K$ is compact in $\mathbb{R}^{n}$, that admits a strong asymptotic expansion of the form

$$
\phi(\mathbf{a}+\mathbf{x})=\phi(\mathbf{a}+r \mathbf{w}) \sim \sum_{j=m}^{\infty} a_{j}(\mathbf{w}) r^{j}, \quad \text { as } \mathbf{x} \rightarrow \mathbf{0},
$$

where $m \in \mathbb{Z}$, and where $a_{j}$ are smooth functions of $\mathbf{w}$, that is, $a_{j} \in \mathcal{D}\left(\mathbb{S}^{n-1}\right)$. We call $\mathcal{D}_{*, \mathbf{a}}\left(\mathbb{R}^{n}\right)$ the space of test functions on $\mathbb{R}^{n}$ with a thick point located at $\mathbf{x}=\mathbf{a}$. It is sometimes convenient to take $\mathbf{a}=\mathbf{0}$; we denote $\mathcal{D}_{*, \mathbf{0}}\left(\mathbb{R}^{n}\right)$ by $\mathcal{D}_{*}\left(\mathbb{R}^{n}\right)$.

Observe that if $\phi$ is a standard test function, namely, smooth in all $\mathbb{R}^{n}$ and with compact support, then it has a Taylor expansion, which may be divergent, but gives 
a strong asymptotic expansion, $\phi(\mathbf{a}+r \mathbf{w}) \sim a_{0}+\sum_{j=1}^{\infty} a_{j}(\mathbf{w}) r^{j}$, where $a_{0}$ is just the real number $\phi(\mathbf{a})$. Hence $\mathcal{D}\left(\mathbb{R}^{n}\right) \subset \mathcal{D}_{*, \mathbf{a}}\left(\mathbb{R}^{n}\right)$; we denote by

$$
i: \mathcal{D}\left(\mathbb{R}^{n}\right) \hookrightarrow \mathcal{D}_{*, \mathbf{a}}\left(\mathbb{R}^{n}\right),
$$

the inclusion map. In fact, with the topology constructed by the following definitions, $\mathcal{D}\left(\mathbb{R}^{n}\right)$ is actually a closed subspace of $\mathcal{D}_{*, \mathbf{a}}\left(\mathbb{R}^{n}\right)$.

The following auxiliary spaces are needed for defining the topoloty of $\mathcal{D}_{*, \mathbf{a}}\left(\mathbb{R}^{n}\right)$

Definition 4. Let $m$ be a fixed integer. The subspace $\mathcal{D}_{*, \mathbf{a}}^{[m]}\left(\mathbb{R}^{n}\right)$ consists of those test functions $\phi$ whose expansion (2.3) begins at $m$. For a fixed compact $K$ whose interior contains $\mathbf{a}, \mathcal{D}_{*, \mathbf{a}}^{[m ; K]}\left(\mathbb{R}^{n}\right)$ is the subspace formed by those test functions of $\mathcal{D}_{*, \mathbf{a}}^{[m]}\left(\mathbb{R}^{n}\right)$ that vanish in $\mathbb{R}^{n} \backslash K$.

Definition 5. Let $m$ be a fixed integer and $K$ a compact subset of $\mathbb{R}^{n}$ whose interior contains $\mathbf{a}$. The topology of $\mathcal{D}_{*, \mathbf{a}}^{[m ; K]}\left(\mathbb{R}^{n}\right)$ is given by the seminorms $\left\{\|\|_{q, s}\right\}_{q>m, s \geq 0}$ defined as

$$
\|\phi\|_{q, s}=\sup _{\mathbf{x}+\mathbf{a} \in K|\mathbf{p}| \leq s} \sup r^{-q}\left|(\partial / \partial \mathbf{x})^{\mathbf{p}} \phi(\mathbf{a}+\mathbf{x})-\sum_{j=m-|\mathbf{p}|}^{q-1} a_{j \cdot \mathbf{p}}(\mathbf{w}) r^{j}\right|,
$$

where $\mathbf{x}=r \mathbf{w}, \mathbf{p} \in \mathbb{N}^{n}$, and $(\partial / \partial \mathbf{x})^{\mathbf{p}} \phi(\mathbf{a}+\mathbf{x}) \sim \sum_{j=m-|\mathbf{p}|}^{\infty} a_{j . \mathbf{p}}(\mathbf{w}) r^{j}$. The topology of $\mathcal{D}_{*, \mathbf{a}}^{[m]}\left(\mathbb{R}^{n}\right)$ is the inductive limit topology of the $\mathcal{D}_{*, \mathbf{a}}^{[m ; K]}\left(\mathbb{R}^{n}\right)$ as $K \nearrow \infty$. The topology of $\mathcal{D}_{*, \mathbf{a}}\left(\mathbb{R}^{n}\right)$ is the inductive limit topology of the $\mathcal{D}_{*, \mathbf{a}}^{[m]}\left(\mathbb{R}^{n}\right)$ as $m \searrow-\infty$.

Next we quote the definition of the "thick distribution space":

Definition 6. The space of distributions on $\mathbb{R}^{n}$ with a thick point at $\mathbf{x}=\mathbf{a}$ is the dual space of $\mathcal{D}_{*, a}\left(\mathbb{R}^{n}\right)$. We denoted it by $\mathcal{D}_{*, a}^{\prime}\left(\mathbb{R}^{n}\right)$, or just as $\mathcal{D}_{*}^{\prime}\left(\mathbb{R}^{n}\right)$ when $\mathbf{a}=\mathbf{0}$. We call the elements of $\mathcal{D}_{*, a}^{\prime}\left(\mathbb{R}^{n}\right)$ "thick distributions".

Let $\pi: \mathcal{D}_{*, a}^{\prime}\left(\mathbb{R}^{n}\right) \rightarrow \mathcal{D}^{\prime}\left(\mathbb{R}^{n}\right)$ denote the projection operator, dual to the inclusion map $i: \mathcal{D}\left(\mathbb{R}^{n}\right) \hookrightarrow \mathcal{D}_{*, \mathbf{a}}\left(\mathbb{R}^{n}\right)$. Since $\mathcal{D}\left(\mathbb{R}^{n}\right)$ is closed in $\mathcal{D}_{*, \mathbf{a}}\left(\mathbb{R}^{n}\right)$, the Hahn-Banach theorem immediately yields that:

Theorem 1. Let $f$ be any distribution in $\mathcal{D}^{\prime}\left(\mathbb{R}^{n}\right)$, then there exists thick distributions $g \in \mathcal{D}_{*, a}^{\prime}\left(\mathbb{R}^{n}\right)$ such that $\pi(g)=f$.

Example 1. (Thick delta functions of degree q) Let $g(\mathbf{w})$ is a distribution in $\mathbb{S}^{n-1}$. The thick delta function of degree $q$, denoted as $g \delta_{*}^{[q]}$, acts on a thick test function $\phi(\mathbf{x})$ as

$$
\left\langle g \delta_{*}^{[q]}, \phi\right\rangle=\frac{1}{C_{n-1}}\left\langle g(\mathbf{w}), a_{q}(\mathbf{w})\right\rangle,
$$

where $\phi(r \mathbf{w}) \sim \sum_{j=m}^{\infty} a_{j}(\mathbf{w}) r^{j}$ as $\mathbf{x} \rightarrow 0$, and $C_{n-1}=\int_{\mathbb{S}^{n-1}} d \sigma(\mathbf{w})$ is the surface area of the n-dimensional unit sphere.

We consider the special case when $q=0, g(\mathbf{w}) \equiv 1$, we denote it $\delta_{*}^{[0]}:=\delta_{*}$. One could easily check that $\pi\left(\delta_{*}\right)=\delta$, is the famous Dirac delta function.

All operations on thick distributions are defined via duality, especially the multiplication and the derivative:

Definition 7. If $\psi \phi \in \mathcal{D}_{*}\left(\mathbb{R}^{n}\right)$ for any $\phi \in \mathcal{D}_{*}\left(\mathbb{R}^{n}\right)$, then for an $f \in \mathcal{D}_{*}^{\prime}\left(\mathbb{R}^{n}\right), \psi f$ is well-defined as $\langle\psi f, \phi\rangle=\langle f, \psi \phi\rangle$. 
Definition 8. Let $f \in \mathcal{D}_{*}^{\prime}\left(\mathbb{R}^{n}\right)$ is a thick distribution, then the thick distributional derivative of $f$ is defined as

$$
\left\langle\frac{\partial^{*} f}{\partial x_{j}}, \phi\right\rangle=-\left\langle f, \frac{\partial \phi}{\partial x_{j}}\right\rangle, \quad \phi \in \mathcal{D}_{*}\left(\mathbb{R}^{n}\right)
$$

One could easily see that $\pi\left(\frac{\partial^{*} f}{\partial x_{j}}\right)=\frac{\bar{\partial} f}{\partial x_{j}}$, the distributional derivative of $f$.

\section{Reconstruction of the OnE-Dimensional CASE}

3.1. Asymptotic expansions with respect to $r$. Now let us view the line segment $[-1,1]$ as 1-dimensional unit ball, and the boundary, i.e., the two points at -1 and 1 as the " 0 dimensional unit sphere". We denote the two boundary points $\mathbf{- 1}$ and $\mathbf{1}$, respectively. We denote the set $\{-\mathbf{1}, \mathbf{1}\}$ as $\mathbb{S}^{0}$, in accordance to the name "0 dimensional unit sphere".

Thus we can generalize the concept of "functions on the unit sphere" to "functions on the 0 dimensional unit sphere": that is, a function from two points to $\mathbb{R}$. Notice that the two points are disconnected, and any functions from one point to $\mathbb{R}$ is just a constant.

Now we can express the $\mathbb{R} \backslash\{0\}$ as $\mathbb{R} \backslash\{0\} \subset \mathbb{S}^{0} \times \mathbb{R}_{\geqslant 0}: x=(\mathbf{w}, r)$, where $r=|x|$; $\mathbf{w}=\mathbf{1}$ when $x>0$ and $\mathbf{w}=-\mathbf{1}$ when $x<0$. That is, if $r>0,(\mathbf{1}, r)$ denotes all positive numbers while $(-\mathbf{1}, r)$ denotes all negative numbers. Notice there are two points in $\mathbb{S}^{0} \times \mathbb{R}_{\geqslant 0}$ with $r=0$, that is, $(\mathbf{1}, 0)$ and $(-\mathbf{1}, 0)$.

Notice that $\mathbb{S}^{0}$ has the natural discrete topology. We endow the space $\mathbb{S}^{0} \times \mathbb{R}_{\geq 0}$ with the product topology. We endow $\mathbb{S}^{0} \times \mathbb{R}_{+}$with the product topology, and it is not hard to see that $\mathbb{R} \backslash\{0\}$ is homeomorphic to $\mathbb{S}^{0} \times \mathbb{R}_{+}$.

Definition 9. Let $r=|x|$, we say a function $f(x)=f(\mathbf{w}, r)$ defined on $\mathbb{R} \backslash\{0\}$ has an asymptotic expansion $\sum_{j=m}^{\infty} a_{j}(\mathbf{w}) r^{j}$, as $x \rightarrow 0$, where $\mathbf{w} \in \mathbb{S}^{0}, a_{i}(\mathbf{w})$ is a function on $\mathbb{S}^{0}$, if

$$
\lim _{r \rightarrow 0^{+}}\left|f(x)-\sum_{j=m}^{M} a_{j}(\mathbf{w}) r^{j}\right| r^{-M}=0, \quad \text { uniformly on } \mathbf{w} \in \mathbb{S}^{0} .
$$

In this case we write $f(x) \sim \sum_{j=m}^{\infty} a_{j}(\mathbf{w}) r^{j}$ as $x \rightarrow 0$. In fact, we can interchange $x \rightarrow 0$ with $r \rightarrow 0^{+}$here.

Let us present a few examples.

Example 2. The first example would be polynomials. Without loss of generality, suppose $f(x)=a_{2 m} x^{2 m}+a_{2 m-1} x^{2 m-1}+\ldots+a_{0}$ is a polynomial of even degree. One could see that $f(x)=f(\mathbf{w}, r)=a_{2 m} r^{2 m}+a_{2 m-1}(\mathbf{w}) r^{2 m-1}+\ldots+a_{0}$, where the coefficients of even power terms $a_{2 k}$ are constants and the coefficients $a_{2 k-1}(\mathbf{w})$ of all odd power terms are functions on $\mathbb{S}^{0}$ :

$$
a_{2 k-1}(\mathbf{w})=\left\{\begin{array}{rl}
a_{2 k-1} & \text { when } \mathbf{w}=\mathbf{1} \\
-a_{2 k-1} & \text { when } \mathbf{w}=-\mathbf{1}
\end{array}, 1 \leq k \leq m .\right.
$$

Clearly, we have the asymptotic expansion $f(\mathbf{w}, r) \sim a_{0}+\ldots+a_{2 m-1}(\mathbf{w}) r^{2 m-1}+$ $a_{2 m} r^{2 m}$ as $x \rightarrow 0$. 
The next example shows the elegance of such description when we try to describe functions with a jump singularity at the origin. Let us present the Heaviside function.

Example 3. The Heaviside function is given as

$$
H(x)=\left\{\begin{array}{ll}
1 & \text { when } x>0 \\
0 & \text { when } x<0
\end{array} .\right.
$$

Let us write it in the above notation, then

$$
H(x)=a_{0}(\mathbf{w})=\left\{\begin{array}{cc}
1 & \text { when } \mathbf{w}=\mathbf{1} \\
0 & \text { when } \mathbf{w}=-\mathbf{1}
\end{array},\right.
$$

and it admits an asymptotic expansion $H(x) \sim a_{0}(\mathbf{w})$ as $x \rightarrow 0$.

Let us present a lemma now concerning the relationship between the asymptotic expansion with respect to $x$ and the asymptotic expansion with respect to $r$ :

Lemma 1. Suppose $f(x): \mathbb{R} \backslash\{0\} \rightarrow \mathbb{R}$ admits an asymptotic expansion $f \sim$ $\sum_{j=m}^{\infty} a_{2 j} x^{2 j}+a_{2 j+1} x^{2 j+1}$ as $x \rightarrow 0$, then it admits an asymptotic expansion $f \sim$ $\sum_{j=m}^{\infty} a_{2 j} r^{2 j}+a_{2 j+1}(\mathbf{w}) r^{2 j+1}$ as $r \rightarrow 0^{+}$. Where $a_{2 j}$ are constants, and

$$
a_{2 j+1}(\mathbf{w})=\left\{\begin{array}{rl}
a_{2 j+1} & \text { when } \mathbf{w}=\mathbf{1} \\
-a_{2 j+1} & \text { when } \mathbf{w}=-\mathbf{1}
\end{array} .\right.
$$

Here we can start the expansion with an even term because we can allow $a_{2 m}=0$.

Proof. Since $f(x)$ admits an asymptotic expansion $f \sim \sum_{j=m}^{\infty} a_{2 j} x^{2 j}+a_{2 j+1} x^{2 j+1}$, then

$$
\lim _{x \rightarrow 0}\left|f(x)-\sum_{j=2 m}^{M} a_{j} x^{j}\right||x|^{-M}=0
$$

On the other hand,

$$
0=\lim _{x \rightarrow 0^{+}}\left|f(x)-\sum_{j=2 m}^{M} a_{j} x^{j}\right||x|^{-M}=\lim _{r \rightarrow 0^{+}}\left|f(r)-\sum_{j=2 m}^{M} a_{j} r^{j}\right| r^{-M}
$$

And

$$
0=\lim _{x \rightarrow 0^{-}}\left|f(x)-\sum_{j=2 m}^{M} a_{j} x^{j}\right||x|^{-M}=\lim _{r \rightarrow 0^{+}}\left|f(-r)-\sum_{j=2 m}^{M} a_{j}(-r)^{j}\right| r^{-M}
$$

Combine(3.3) and (3.4), we have

$$
\lim _{r \rightarrow 0^{+}}\left|f(x)-\sum_{j=2 m}^{M} a_{j}(\mathbf{w}) r^{j}\right| r^{-M}=0,
$$

where $a_{j}(\mathbf{w})=a_{j}$ when $j$ is even, and

$$
a_{j}(\mathbf{w})=\left\{\begin{aligned}
a_{j} & \text { when } \mathbf{w}=\mathbf{1} \\
-a_{j} & \text { when } \mathbf{w}=-\mathbf{1}
\end{aligned}\right.
$$

when $j$ is odd. Thus by definition 9, we obtain the statement of the lemma. 
Example 4. Let $f: \mathbb{R} \rightarrow \mathbb{R}$ be a smooth function. It has a Taylor expansion at the origin: $f(x) \sim \sum_{j=0}^{\infty} \frac{f^{(j)}(0)}{j !} x^{j}$. It may not converge. But it is an asymptotic expansion as $x \rightarrow 0$. By the Lemma 1, we have an asymptotic expansion

$$
f \sim \sum_{j=0}^{\infty} a_{2 j} r^{2 j}+a_{2 j+1}(\mathbf{w}) r^{2 j+1} \quad \text { as } r \rightarrow 0^{+},
$$

where $a_{2 j}=\frac{f^{(2 j)}(0)}{(2 j) !}$ and

$$
a_{2 j+1}(\mathbf{w})=\left\{\begin{array}{rl}
\frac{f^{(2 j+1)}(0)}{(2 j+1) !} & \text { when } \mathbf{w}=\mathbf{1} \\
-\frac{f^{(2 j+1)}(0)}{(2 j+1) !} & \text { when } \mathbf{w}=-\mathbf{1}
\end{array} .\right.
$$

Next we want to discuss the derivative of $\sum_{j=m}^{\infty} a_{j}(\mathbf{w}) r^{j}$ with respect to $x$. We first discuss $\frac{d\left(a(\mathbf{w}) r^{j}\right)}{d x}$, where $\mathbf{w} \in \mathbb{S}^{0}, a(\mathbf{w})$ is a function on $\mathbb{S}^{0}$. Because there is a natural inclusion $\mathbb{R} \backslash\{0\} \subset \mathbb{S}^{0} \times \mathbb{R}_{\geqslant 0}$, clearly, $a(\mathbf{w}) r^{j}$ can be viewed as a function on $x$ when $r=|x| \neq 0$. Thus, it is legal to talk about "derivative with respect to $\mathrm{x}$ " in the usual sense.

We first discuss the derivative $\frac{d\left(a(\mathbf{w}) r^{j}\right)}{d x}, j \neq 0$, at $x_{0}>0$. Denote the coordinate of $x_{0}$ at $\mathbb{S}^{0} \times \mathbb{R}_{\geq 0}$ as $\left(\mathbf{1}, x_{0}\right)$. Since $\mathbb{S}^{0}$ is endowed with the discrete topology, there is a small neighborhood of $\left(\mathbf{1}, x_{0}\right)$, denoted as $\{\mathbf{1}\} \times\left[x_{0}-\delta, x_{0}+\delta\right]$, on which the function $a(\mathbf{w}) r^{j}$ equals $a(\mathbf{1}) x^{j}$, where $a(\mathbf{1})$ is the value of the function $a(\mathbf{w})$ at $\mathbf{w}=\mathbf{1}$, namely, a constant. Thus we have

$$
\left.\frac{d\left(a(\mathbf{w}) r^{j}\right)}{d x}\right|_{x=x_{0}}=\left.\frac{d\left(a(\mathbf{1}) x^{j}\right)}{d x}\right|_{x=x_{0}}=\left.a(\mathbf{1}) j x^{j-1}\right|_{x=x_{0}} .
$$

Similarly, when $x_{0}<0$, there is a small neighborhood of $\left(-\mathbf{1},-x_{0}\right)$, denoted as $\{-\mathbf{1}\} \times\left[-x_{0}-\delta,-x_{0}+\delta\right]$, on which the function $a(\mathbf{w}) r^{j}$ equals $a(-\mathbf{1})(-x)^{j}$, hence when $x_{0}<0, j \neq 0$, we have

$$
\left.\frac{d\left(a(\mathbf{w}) r^{j}\right)}{d x}\right|_{x=x_{0}}=-\left.a(-\mathbf{1}) j(-x)^{j-1}\right|_{x=x_{0}} .
$$

When $j=0$, a similar analysis shows that $\left.\frac{d(a(\mathbf{w}))}{d x}\right|_{x=x_{0}}=0$ when $x_{0} \neq 0$.

When $x=0, a(\mathbf{w})$ can be viewed as a multi-valued function on $x$. On the other hand, if $j>0$, then $a(\mathbf{w}) r^{j}=0$ at $x=0$. We can talk about the so called "leftderivative" and "right-derivative" of $a(\mathbf{w}) r^{j}, j>0$ at $x=0$. It is not hard to see, the "left-derivative" and "right-derivative" are both 0 when $j>1$; and the "right-derivative" equals $a(\mathbf{1})$ while the "left-derivative" equals $a(-\mathbf{1})$ when $j=1$, at $x=0$.

Based on the above discussion, we have the following theorem regarding the derivative $\frac{d\left(a(\mathbf{w}) r^{j}\right)}{d x}$ :

Theorem 2. Let $a(\mathbf{w}) r^{j}$ be a function on $\mathbb{S}^{0} \times \mathbb{R}_{+}$, then

$$
\frac{d\left(a(\mathbf{w}) r^{j}\right)}{d x}=\left\{\begin{array}{c}
a(\mathbf{1}) j x^{j-1} \quad \text { when } x>0 \\
-a(-\mathbf{1}) j(-x)^{j-1} \text { when } x<0
\end{array} .\right.
$$


In summary, it amounts to saying $\frac{d\left(a(\mathbf{w}) r^{j}\right)}{d x}=b(\mathbf{w}) r^{j-1}$, where $b(\mathbf{w})$ is a function on $\mathbb{S}^{0}$ :

$$
b(\mathbf{w})=\left\{\begin{array}{cl}
a(\mathbf{1}) j & \text { when } \mathbf{w}=\mathbf{1} \\
-a(-\mathbf{1}) j & \text { when } \mathbf{w}=-\mathbf{1}
\end{array} .\right.
$$

We immediately have the following theorem:

Theorem 3. If $f(x) \sim \sum_{j=m}^{\infty} a_{j}(\mathbf{w}) r^{j}$ as $x \rightarrow 0$, then the term-by-term derivative with respect to $x$ of the expasion takes the following form in $\mathbb{S}^{0} \times \mathbb{R}_{+}$:

$$
\begin{aligned}
\sum_{j=m}^{\infty} \frac{d\left(a_{j}(\mathbf{w}) r^{j}\right)}{d x} & =\sum_{j=m-1}^{\infty} a_{j, 1}(\mathbf{w}) r^{j}, \\
\text { where } a_{j, 1}(\mathbf{w}) & =\left\{\begin{array}{cc}
a_{j+1}(\mathbf{w})(j+1) & \text { when } \mathbf{w}=\mathbf{1} \\
-a_{j+1}(\mathbf{w})(j+1) & \text { when } \mathbf{w}=-\mathbf{1}
\end{array}\right.
\end{aligned}
$$

Example 5. The above theorems show that the usual derivative (NOT the distributional derivative) of the Heaviside function is 0 on $\mathbb{S}^{0} \times \mathbb{R}_{+}=\mathbb{R} \backslash\{0\}$. Here we can clearly distinguish between the "usual derivative" and the "distributional derivative". Since we know the famous fact that the "distributinal derivative" of the Heaviside function is the Dirac delta function.

Now we are ready to construct the space of thick test functions.

\subsection{Reconstruction of the space of test functions on $\mathbb{R}$ with a thick point.}

Definition 10. Let $\phi \in C^{\infty}(\mathbb{R} \backslash\{0\})$. We say that $\phi(x)$ has a "strong" expansion $\sim \sum_{j=m}^{\infty} a_{j}(\mathbf{w}) r^{j}$ as $x \rightarrow 0$ if the asymptotic development of $\frac{d^{p} \phi}{d x^{p}}$ exists and equals the term-by-term differentiation $\sum_{j=m}^{\infty} \frac{d^{p}\left(a_{j}(\mathbf{w}) r^{j}\right)}{d x^{p}}$, as the derivative was discussed in the previous section.

We now define the space of test functions with a thick point at $x=a$.

Definition 11. Let $\mathcal{D}_{*, a}(\mathbb{R})$ denote the vector space of all compactly supported smooth functions $\phi$ defined in $\mathbb{R} \backslash\{a\}$, that admit a strong asymptotic expansion of the form

$$
\phi(a+x) \sim \sum_{j=m}^{\infty} a_{j}(\mathbf{w}) r^{j}, \quad \text { as } x \rightarrow 0 .
$$

where $a_{j}(\mathbf{w})$ is a function on $\mathbb{S}^{0}$ as defined above in definition 9 . We call $\mathcal{D}_{*, a}(\mathbb{R})$

"the space of test functions on $\mathbb{R}$ with a thick point located at $x=a$ ". We denote $\mathcal{D}_{*, 0}(\mathbb{R})$ as $\mathcal{D}_{*}(\mathbb{R})$.

The definition of $\mathcal{D}_{*, a}(\mathbb{R})$ is very much analogous to the defintion of $\mathcal{D}_{*, a}\left(\mathbb{R}^{n}\right)$ when $n \geq 2$. [27]

On the other hand, the thick test functions defined in [7 is included in the above definition. Recall that a thick test function defined in [7] is a compactly supported function $\phi$ with domain $\mathbb{R}$, smooth in $\mathbb{R} \backslash\{a\}$, and at $x=a$ all its onesided derivatives,

$$
\phi^{(n)}(a \pm 0)=\lim _{x \rightarrow a^{ \pm}} \phi^{(n)}(x), \quad \forall n \in \mathbb{N}
$$


exist. Here let us introduce a different notation $\mathcal{D}_{*, a}^{\text {old }}(\mathbb{R})$ to denote the space of such functions. One can see that any function in $\mathcal{D}_{*, a}^{\text {old }}(\mathbb{R})$ admits a strong asymptotic expansion

$$
\begin{gathered}
\phi(a+x) \sim \sum_{j=0}^{\infty} a_{j}(\mathbf{w}) r^{j}, \quad \text { as } x \rightarrow 0, \\
\text { where } a_{j}(\mathbf{w})=\left\{\begin{array}{cc}
\frac{\phi^{(j)}(a+0)}{j !} & \text { when } \mathbf{w}=\mathbf{1} \\
(-1)^{j} \frac{\phi^{(j)}(a-0)}{j !} & \text { when } \mathbf{w}=-\mathbf{1}
\end{array} .\right.
\end{gathered}
$$

In particular, if $\phi(x)$ has a jump discontinuity at $x=a$, then in the expansion (3.8), $a_{0}(\mathbf{1}) \neq a_{0}(-\mathbf{1})$.

By example 4, all compactly supported smooth functions form a closed subspace of $\mathcal{D}_{*, a}^{\text {old }}(\mathbb{R})$, thus there are natural inclusion maps :

$$
\mathcal{D}(\mathbb{R}) \hookrightarrow \mathcal{D}_{*, a}^{\text {old }}(\mathbb{R}) \hookrightarrow \mathcal{D}_{*, a}(\mathbb{R}) .
$$

Now let us define a topology on $\mathcal{D}_{*, a}(\mathbb{R})$ and make it a topological vector space. Similar to [27, we denote the subspace $\mathcal{D}_{*, a}^{[m]}(\mathbb{R})$ as the test functions $\phi$ whose expansion (3.7) begins at $m$. For a fixed compact $K$ whose interior contains $a, \mathcal{D}_{*, a}^{[m, K]}(\mathbb{R})$ is the subspace formed by those test functions of $\mathcal{D}_{*, a}^{[m]}(\mathbb{R})$ that vanish in $\mathbb{R} \backslash K$.

Now let us define the topology of the space of thick test functions in $\mathbb{R}$.

Definition 12. Let $m$ be a fixed integer and $K$ a compact subset of $\mathbb{R}$ whose interior contains a. The topology of $\mathcal{D}_{*, a}^{[m, K]}(\mathbb{R})$ is given by the seminorms $\left\{\|\|_{q, s}\right\}_{s \geq 0}$ defined

$$
\|\phi\|_{q, s}=\sup _{x+a \in K 0 \leq p \leq s} \sup r^{-q}\left|(d / d x)^{p} \phi(a+x)-\sum_{j=m-p}^{q-1} a_{j, p}(\mathbf{w}) r^{j}\right|,
$$

where $(d / d x)^{p} \phi(a+x) \sim \sum_{j=m-p}^{q-1} a_{j, p}(\mathbf{w}) r^{j}$. The topology of $\mathcal{D}_{*, a}^{[m]}(\mathbb{R})$ is the inductive limit topology of the $\mathcal{D}_{*, a}^{[m, K]}(\mathbb{R})$ as $K \nearrow \infty$. The topology of $\mathcal{D}_{*, a}(\mathbb{R})$ is the inductive limit topology of $\mathcal{D}_{*, a}^{[m]}(\mathbb{R})$ as $m \searrow-\infty$.

One could see that the "space of test functions in $\mathbb{R}$ " that is introduced in the paper [7] is a closed subspace of $\mathcal{D}_{*, a}(\mathbb{R})$ introduced above: $\mathcal{D}_{*, a}^{\text {old }}(\mathbb{R}) \subseteq \mathcal{D}_{*, a}(\mathbb{R})$. Moreover, $\mathcal{D}_{*, a}^{\text {old }}(\mathbb{R})$ is closed in $\mathcal{D}_{*, a}(\mathbb{R})$ with respect to derivatives.

Furthermore, there is a natural inclusion map $i: \mathcal{D}(\mathbb{R}) \hookrightarrow \mathcal{D}_{*, a}(\mathbb{R})$. With the topology defined in definition 12 the space of usual test functions $\mathcal{D}(\mathbb{R})$ is a closed subspace of $\mathcal{D}_{*, a}(\mathbb{R})$, similar to the case of higher dimensions [27].

3.3. Space of distributions on $\mathbb{R}$ with a thick point. Having all the preparation above, we can define distributions on $\mathbb{R}$ with a thick point.

Definition 13. The space of distributions on $\mathbb{R}$ with a thick point at $x=a$ is the dual space of $\mathcal{D}_{*, a}(\mathbb{R})$. We denote it $\mathcal{D}_{*, a}^{\prime}(\mathbb{R})$, or just as $\mathcal{D}_{*}^{\prime}(\mathbb{R})$ when $a=0$. We call the elements of $\mathcal{D}_{*, a}^{\prime}(\mathbb{R})$ "thick distributions". 
Let $\pi: \mathcal{D}_{*, a}^{\prime}(\mathbb{R}) \rightarrow \mathcal{D}^{\prime}(\mathbb{R})$, be the projection operator, dual to the inclusion $i: \mathcal{D}(\mathbb{R}) \hookrightarrow \mathcal{D}_{*, a}(\mathbb{R})$. Since $\mathcal{D}(\mathbb{R})$ is closed in $\mathcal{D}_{*, a}(\mathbb{R})$, by the Hanh-Banach theorem we have the following result.

Theorem 4. Let $f$ be any distribution in $\mathcal{D}^{\prime}(\mathbb{R})$, then there exist thick distributions $g \in \mathcal{D}_{*, a}^{\prime}(\mathbb{R})$ such that $\pi(g)=f$.

Naturally, if $f \in \mathcal{D}^{\prime}(\mathbb{R})$ then there are infinitely many thick distributions $g$ with $\pi(g)=f$.

Before giving any examples of thick distributions, let us recall that by the convention of the discrete measure, the "integral" of a function on a discrete set is just the summation of the function over these discrete points. Using our notation in this present article, one just write, for any function $\phi$ defined on $\mathbb{S}^{0}$ :

$$
\int_{S^{0}} \phi(\mathbf{w}) d \sigma(\mathbf{w})=\phi(\mathbf{1})+\phi(-\mathbf{1}) .
$$

For example, for the Heaviside function as in equation (3.2): $H(x)=a_{0}(\mathbf{w})$,

$$
\int_{S^{0}} a_{0}(\mathbf{w}) d \sigma(\mathbf{w})=1
$$

For a constant function $\phi(\mathbf{w}) \equiv 1$,

$$
\int_{S^{0}} 1 d \sigma(\mathbf{w})=\int_{S^{0}} d \sigma(\mathbf{w})=2 .
$$

Thus one can discuss the "double integral" on $\mathbb{S}^{0} \times \mathbb{R}_{+}$if it exists:

$$
\begin{aligned}
\int_{S^{0}} \int_{0}^{+\infty} \phi(\mathbf{w}, r) d r d \sigma(\mathbf{w}) & =\int_{0}^{+\infty}[\phi(\mathbf{1}, r)+\phi(-\mathbf{1}, r)] d r \\
& =\int_{0}^{+\infty} \phi(x) d x+\int_{0}^{-\infty} \phi(x) d(-x) \\
& =\int_{0}^{+\infty} \phi(x) d x+\int_{-\infty}^{0} \phi(x) d x .
\end{aligned}
$$

Clearly, if $\phi \in \mathcal{D}(\mathbb{R})$ is a usual test function, then

$$
\int_{S^{0}} \int_{0}^{+\infty} \phi(\mathbf{w}, r) d r d \sigma(\mathbf{w})=\int_{-\infty}^{+\infty} \phi(x) d x
$$

is just a normal integral over $\mathbb{R}$.

Next let us present a few examples of thick distributions in $\mathcal{D}_{*, a}^{\prime}(\mathbb{R})$.

It is well known that any locally integrable function $f$ defined in $\mathbb{R}$ yields a distribution, usually denoted by the same notation $f$, by the prescription

$$
\langle f, \phi\rangle=\int_{-\infty}^{+\infty} f(x) \phi(x) d x, \quad \phi \in \mathcal{D}(R) .
$$

Similar to the higher dimensional case, if $a \notin \operatorname{supp} f$, that is, if $f(x)=0$ for $|x-a|<\epsilon$ for some $\epsilon>0$, then (3.13) will also work in $\mathcal{D}_{*, a}^{\prime}(\mathbb{R})$. However, if $a \in \operatorname{supp} f$, then, in general the integral $\int_{-\infty}^{+\infty} f(x) \phi(x) d x$ would be divergent and thus a thick distribution that one could call " $f$ " cannot be defined in a canonical way. Nevertheless, it is possible in many cases to define a "finite part" distribution $P f(f(x))$ which is the canonical thick distribution corresponding to $f$. Let us recall at this point the definition of the finite part of a limit [8]. 
Definition 14. Let $F$ be a function defined in an interval of the form $(0, a)$ for some $a>0$. We say that the finite part of the limit of $F(\epsilon)$ as $\epsilon \rightarrow 0^{+}$exists and equals $A$, and denote this as F.p. $\lim _{\epsilon \rightarrow 0^{+}} F(\epsilon)=A$, if $F$ has the decomposition $F(\epsilon)=$ $F_{\text {fin }}(\epsilon)+F_{\text {infin }}(\epsilon)$, where the infinite part $F_{\text {infin }}(\epsilon)$ is a finite linear combination of functions of the type $\epsilon^{-p} \ln ^{q} \epsilon$, where $p \geq 0$ and $q>0$, and where the finite part $F_{\text {fin }}(\epsilon)$ is a function whose limit as $\epsilon \rightarrow 0^{+}$equals $A$.

Definition 15. Let $f$ be a locally integrable function definied in $\mathbb{R} \backslash\{a\}$. The thick distribution $\operatorname{Pf}(f(x))$ is defined as

$$
\begin{aligned}
\langle P f(f(x)), \phi(x)\rangle & =F . p . \int_{-\infty}^{+\infty} f(x) \phi(x) d x \\
& =F . p . \lim _{\epsilon \rightarrow 0^{+}} \int_{|x-a| \geq \epsilon} f(x) \phi(x) d x, \quad \phi \in \mathcal{D}_{*, a}(\mathbb{R}),
\end{aligned}
$$

provided that the finite part integrals exist for all $\phi \in \mathcal{D}_{*, a}(\mathbb{R})$. Here if we set $x-a=y,|y|=r$, then

$$
\begin{aligned}
\int_{|x-a| \geq \epsilon} f(x) \phi(x) d x & =\int_{|y| \geq \epsilon} f(y+a) \phi(y+a) d y=\int_{|y| \geq \epsilon} g(y) \psi(y) d y \\
& =\int_{S^{0}} \int_{\epsilon}^{+\infty} g(\mathbf{w}, r) \psi(\mathbf{w}, r) d r \\
& =\int_{\epsilon}^{+\infty} g(\mathbf{1}, r) \psi(\mathbf{1}, r) d r+\int_{\epsilon}^{+\infty} g(-\mathbf{1}, r) \psi(-\mathbf{1}, r) d r \\
& =\int_{\epsilon}^{+\infty} g(y) \psi(y) d y+\int_{-\epsilon}^{-\infty} g(y) \psi(y) d(-y) \\
& =\int_{a+\epsilon}^{+\infty} f(x) \phi(x) d x+\int_{-\infty}^{a-\epsilon} f(x) \phi(x) d x .
\end{aligned}
$$

Similar to the higher dimensional case, although the finite part limit is not defined for all locally integrable functions $f, P f(f(x))$ is defined in many important and interesting cases.

Example 6. If $\lambda \in \mathbb{C}$ then Pf $\left(|x-a|^{\lambda}\right)$ is a well-defined thick distribution of $\mathcal{D}_{*, a}^{\prime}(\mathbb{R})$. Indeed, one needs to consider the finite part of the integral $\int_{-\infty}^{+\infty}|x-a|^{\lambda} \phi(x) d x$ for any $\phi(x) \in \mathcal{D}_{*, a}(\mathbb{R})$. Explicitly, since F.p. $\int_{0}^{A} r^{\alpha} d r=A^{\alpha+1} /(\alpha+1), \alpha \neq$ $-1, F . p . \int_{0}^{A} r^{-1} d r=\log A$, we obtain that if $\lambda \notin \mathbb{Z}$ then

$$
\begin{aligned}
\left\langle\operatorname{Pf}\left(|x-a|^{\lambda}\right), \phi(x)\right\rangle & =\int_{|x-a| \geq A}|x-a|^{\lambda} \phi(x) d x \\
& +\int_{|x-a|<A}|x-a|^{\lambda}\left(\phi(x)-\sum_{j \leq-\operatorname{Re} \lambda-1} a_{j}(w)|x-a|^{j}\right) d x \\
& +\sum_{j \leq-\operatorname{Re} \lambda-1}\left(a_{j}(\mathbf{1})+a_{j}(-\mathbf{1})\right) \frac{A^{\lambda+j+1}}{\lambda+j+1},
\end{aligned}
$$


while if $\lambda=k \in \mathbb{Z}$ then

$$
\begin{aligned}
\left\langle\operatorname{Pf}\left(|x-a|^{\lambda}\right), \phi(x)\right\rangle & =\int_{|x-a| \geq A}|x-a|^{k} \phi(x) d x \\
& +\int_{|x-a|<A}|x-a|^{k}\left(\phi(x)-\sum_{j \leq-k-1} a_{j}(w)|x-a|^{j}\right) d x \\
& +\sum_{j<-k-1}\left(a_{j}(\mathbf{1})+a_{j}(-\mathbf{1})\right) \frac{A^{\lambda+j+1}}{\lambda+j+1}+\left(a_{-k-1}(\mathbf{1})+a_{-k-1}(-\mathbf{1})\right) \log A .
\end{aligned}
$$

Formulas (3.16) and (3.17) hold for any $A>0$. The finite part is needed for all $\lambda$ in the space of thick distributions $\mathcal{D}_{*, a}^{\prime}(\mathbb{R})$.

Using the ideas of the previous example one can show that when $\psi$ is smooth in all of $\mathbb{R}$, i.e., $\psi \in \mathcal{E}(\mathbb{R})$, then $\operatorname{Pf}(\psi(x)) \in \mathcal{D}_{*, a}^{\prime}(\mathbb{R})$; notice that the finite part regularization is always needed if there is a thick point in the support of $\psi$.

Example 7. This example will be the "finite part regularization" of the Heaviside function. The regulariztion is needed because of the singularity of the thick test functions: let $\phi(x) \in \mathcal{D}_{*}(\mathbb{R})$, the $\operatorname{Pf}(H(x)) \in \mathcal{D}_{*}^{\prime}(\mathbb{R})$ is defined as:

$$
\begin{aligned}
\langle P f(H(x)), \phi(x)\rangle & =\int_{|x-a| \geq A} H(x) \phi(x) d x+\int_{|x-a|<A} H(x)\left(\phi(x)-\sum_{j \leq-1} a_{j}(w) r^{j}\right) d x \\
& +\sum_{j<-1} a_{j}(\mathbf{1}) \frac{A^{j+1}}{j+1}+a_{-1}(\mathbf{1}) \log A .
\end{aligned}
$$

Similar to the higher dimensional case, we can also define a "thick delta function with degree q". This definition is closely related to the extension of Dirac delta function introduce in 7 . Note that here we use the notation $C_{0}=\int_{S^{0}} 1 d \sigma(\mathbf{w})=$ $\int_{S^{0}} d \sigma(\mathbf{w})=2$.

Definition 16. Let $g(\mathbf{w})$ be a distribution in $\mathbb{S}^{0}$, the thick delta function of degree $q$, denoted as $g \delta_{*}^{[q]}$, acts on a thick test function $\phi(x)$ as

$$
\left\langle g \delta_{*}^{[q]}, \phi\right\rangle_{\mathcal{D}_{*}^{\prime}(\mathbb{R}) \times \mathcal{D}_{*}(\mathbb{R})}=\frac{1}{C_{0}}\left\langle g(\mathbf{w}), a_{q}(\mathbf{w})\right\rangle_{\mathcal{D}_{*}^{\prime}(\mathbb{S}) \times \mathcal{D}_{*}(\mathbb{S})},
$$

where $\phi(x) \sim \sum_{j=m}^{\infty} a_{j}(\mathbf{w}) r^{j}$, as $x \rightarrow 0$, and $C_{0}=2$.

The thick delta function of degree 0 , namely $g \delta_{*}^{[0]}$ will be denoted as $g \delta_{*}$, as $g(\mathbf{w}) \delta_{*}$, or as $g(\mathbf{w}) \delta_{*}(x)$. In particular, if $g(x) \equiv 1$, then we obtain the onedimensional "plain thick delta function" $\delta_{*}$, given as

$$
\left\langle\delta_{*}, \phi\right\rangle_{\mathcal{D}_{*}^{\prime}(\mathbb{R}) \times \mathcal{D}_{*}(\mathbb{R})}=\frac{1}{C_{0}} \int_{\mathbb{S}^{0}} a_{0}(\mathbf{w}) d \sigma(\mathbf{w})=\frac{a_{0}(\mathbf{1})}{2}+\frac{a_{0}(-\mathbf{1})}{2} .
$$

Remark 1. If $\phi \in \mathcal{D}(\mathbb{R})$ is a usual test function, then by example 4 we see that

$$
\left\langle\pi\left(\delta_{*}\right), \phi\right\rangle=\left\langle\delta_{*}, i(\phi)\right\rangle=\frac{\phi(0)}{2}+\frac{\phi(0)}{2}=\phi(0),
$$


hence $\pi\left(\delta_{*}\right)=\delta$.

Moreover, if $\phi \in \mathcal{D}_{*}^{\text {old }}(\mathbb{R})$, let $\pi^{\prime}: \mathcal{D}_{*}^{\prime}(\mathbb{R}) \rightarrow \mathcal{D}_{*}^{\prime o l d}(\mathbb{R})$ be the projection onto the space of thick distributions defined in $\left[7\right.$, let $\phi_{+}(0)$ denote $\lim _{x \rightarrow 0^{+}} \phi(x)$ and $\phi_{-}(0)$ denote $\lim _{x \rightarrow 0^{-}} \phi(x)$. Then by equation (3.8), one can see that

$$
\begin{aligned}
\left\langle\pi^{\prime}\left(\delta_{*}\right), \phi\right\rangle & =\frac{1}{2} \phi_{+}(0)+\frac{1}{2} \phi_{-}(0) \\
& =\langle\widetilde{\delta}(x), \phi(x)\rangle,
\end{aligned}
$$

where $\widetilde{\delta}(x)$ is defined in [7], equation (3.10).

Example 8. Let $g_{\lambda}(\mathbf{w})$ be a distribution in $\mathbb{S}^{0}:\left\langle g_{\lambda}(\mathbf{w}), a(\mathbf{w})\right\rangle=2 \lambda a(\mathbf{1})+$ $2(1-\lambda) a(-\mathbf{1})$, where $0 \leq \lambda \leq 1$ is a constant. Then

$$
\left\langle g_{\lambda} \delta_{*}^{[q]}, \phi\right\rangle_{\mathcal{D}_{*}^{\prime}(\mathbb{R}) \times \mathcal{D}_{*}(\mathbb{R})}=\lambda a_{q}(\mathbf{1})+(1-\lambda) a_{q}(-\mathbf{1}) .
$$

In particular, if $\lambda=1$,

$$
\left\langle g_{1} \delta_{*}^{[q]}, \phi\right\rangle_{\mathcal{D}_{*}^{\prime}(\mathbb{R}) \times \mathcal{D}_{*}(\mathbb{R})}=a_{q}(\mathbf{1}) .
$$

If $\phi \in \mathcal{D}_{*}^{\text {old }}(\mathbb{R})$, then

$$
\left\langle\pi^{\prime}\left(g_{1} \delta_{*}\right), \phi\right\rangle=\phi_{+}(0) .
$$

3.4. Algebraic and analytic operations in $\mathcal{D}_{*, a}^{\prime}(\mathbb{R})$. Naturally, we define the algebraic and anlytic operations in $\mathcal{D}_{*, a}^{\prime}(\mathbb{R})$ in the same way they are defined for the usual distributions, namely, by duality.

3.4.1. Basic definitions. Let $f, g \in \mathcal{D}_{*, a}^{\prime}(\mathbb{R}), \phi(x) \in \mathcal{D}_{*, a}(\mathbb{R})$, and $\lambda \in \mathbb{C}$. Then $f+\lambda g \in \mathcal{D}_{*, a}^{\prime}(\mathbb{R})$ is given as

$$
\langle f+\lambda g, \phi\rangle=\langle f, \phi\rangle+\lambda\langle g, \phi\rangle .
$$

Let $c \in \mathbb{R}$, translations are handled by

$$
\langle f(x+c), \phi(x)\rangle=\langle f(x), \phi(x-c)\rangle .
$$

Notice that $f \in \mathcal{D}_{*, a}^{\prime}(\mathbb{R})$ while the translation $f(x+c) \in \mathcal{D}_{*, a-c}^{\prime}(\mathbb{R})$. Observe that any distribution $g$ of the space $\mathcal{D}_{*, a}^{\prime}(\mathbb{R})$ can be written as $g(x)=f(x-a)$ for some $f \in \mathcal{D}_{*}^{\prime}(\mathbb{R})$, and this justifies studying most results in $\mathcal{D}_{*}^{\prime}(\mathbb{R})$ only.

Moreover,

$$
\langle f(c x), \phi(x)\rangle=\frac{1}{|c|}\langle f(x), \phi(x / c)\rangle .
$$

3.4.2. Multiplication. The operation of multiplication is defined by duality: suppose for any $\phi \in \mathcal{D}_{*, a}(\mathbb{R}), \psi \phi$ is still an element in $\mathcal{D}_{*, a}(\mathbb{R})$. Then if $\rho \in \mathcal{D}_{*, a}^{\prime}(\mathbb{R})$, we define

$$
\langle\psi \rho, \phi\rangle:=\langle\rho, \psi \phi\rangle .
$$

$\psi$ is called a "multiplier" of $\mathcal{D}_{*, a}(\mathbb{R})$ and $\mathcal{D}_{*, a}^{\prime}(\mathbb{R})$.

From the definition one can see that the space of multipliers for a space of test functions and for its dual space are the same, their Moyal algebra. The space of multipliers of the spaces of standard test functions and standard distributions $\mathcal{D}(\mathbb{R})$ and $\mathcal{D}^{\prime}(\mathbb{R})$ is the space $\mathcal{E}(\mathbb{R})$ of all smooth function in $\mathbb{R}$. 
Definition 17. A function $\psi$ defined in $\mathbb{R} \backslash\{a\}$ belongs to $\mathcal{E}_{*, a}(\mathbb{R})$ if $\psi$ is smooth in $\mathbb{R} \backslash\{a\}$ and if for each ordinary test function $\rho \in \mathcal{D}(\mathbb{R})$ the product $\rho \psi$ belongs to $\mathcal{D}_{*, a}(\mathbb{R})$.

Clearly, $\mathcal{E}_{*, a}(\mathbb{R})$ contains all functions that are smooth in $\mathbb{R} \backslash\{a\}$ and having an asymptotic expansion $\sum_{j=m}^{\infty} a_{j}(\mathbf{w}) r^{j}$ as $x \rightarrow a$. Now we show that $\mathcal{E}_{*, a}(\mathbb{R})$ actually is consisted by such functions. Indeed, if we pick $\rho \in \mathcal{D}(\mathbb{R})$ such that $\rho(x) \equiv 1$ in a neighbourhood of $a$, then we see that each $\psi \in \mathcal{E}_{*, a}(\mathbb{R})$ actually admits and expansion $\sum_{j=m}^{\infty} a_{j}(\mathbf{w}) r^{j}$ as $x \rightarrow a$.

It is clear that we have the following proposition.

Proposition 1. The Moyal algebra of $\mathcal{D}_{*, a}(\mathbb{R})$ and of $\mathcal{D}_{*, a}^{\prime}(\mathbb{R})$, namely the space of multipliers, is $\mathcal{E}_{*, a}(\mathbb{R})$.

Example 9. The Heaviside function $H(x)=a_{0}(\mathbf{w})=\left\{\begin{array}{cc}1 & \text { when } \mathbf{w}=\mathbf{1} \\ 0 & \text { when } \mathbf{w}=-\mathbf{1}\end{array}\right.$ is NOT a multiplier of $D^{\prime}(R)$, but it is a multiplier of $D_{*}^{\prime}(R)$.

3.4.3. Derivatives of thick distributions. The derivatives of thick distributions are defined also by duality.

Definition 18. If $f \in \mathcal{D}_{*, a}^{\prime}(\mathbb{R})$ then its thick distributional derivative $d^{*} f / d x$ is defined as

$$
\left\langle\frac{d^{*} f}{d x}, \phi\right\rangle=-\left\langle f, \frac{d \phi}{d x}\right\rangle, \quad \phi \in D_{*, a}(R) .
$$

From the discussion in the previous sections, one sees that in general the spaces $D_{*, a}^{[m]}(R)$ are not closed under differentiation. But $D_{*, a}^{[0]}(R)$ is closed under differentiations.

About the notation: $d / d x$ means the ordinary derivative, $\bar{d} / d x$ means the distributional derivative, $d^{*} / d x$ means the thick distributional derivative. Similar to the higher dimensional case, let $f \in \mathcal{D}_{*, a}^{\prime}(\mathbb{R})$ and $g=\pi(f)$, we have:

$$
\begin{aligned}
\left\langle\pi\left(\frac{d^{*} f}{d x}\right), \phi\right\rangle & =\left\langle\frac{d^{*} f}{d x}, i(\phi)\right\rangle=-\left\langle f, \frac{d i(\phi)}{d x}\right\rangle \\
& =-\left\langle f, i\left(\frac{d \phi}{d x}\right)\right\rangle=-\left\langle\pi(f), \frac{d \phi}{d x}\right\rangle \\
& =\left\langle\frac{\bar{d} g}{d x}, \phi\right\rangle .
\end{aligned}
$$

Hence we obtain the following proposition:

Proposition 2. Let $f \in \mathcal{D}_{*, a}^{\prime}(\mathbb{R})$, then

$$
\pi\left(\frac{d^{*} f}{d x}\right)=\frac{\bar{d} \pi(f)}{d x}
$$

Because the derivative of test functions satisfies the product rule, the derivative of thick distrubutions also satisfy the product rule:

$$
\frac{d^{*}(\psi f)}{d x}=\frac{d \psi}{d x} f+\psi \frac{d^{*} f}{d x}, \quad f \in \mathcal{D}_{*, a}^{\prime}(\mathbb{R}), \psi \in \mathcal{E}_{*, a}(\mathbb{R}) .
$$


Next let us compute a very important example of thick derivatives, the thick derivative of the Heaviside function.

Example 10. The Heaviside function in $\mathcal{D}_{*}^{\prime}(\mathbb{R})$ is defined in the example $\square$, now let us compute the derivative of it. By definition, for any $\phi \in \mathcal{D}_{*}(\mathbb{R})$

$$
\left\langle\frac{d^{*}(P f(H(x)))}{d x}, \phi\right\rangle=-\left\langle P f(H(x)), \frac{d \phi}{d x}\right\rangle .
$$

Now suppose $d \phi / d x$ has asymptotic expansion $d \phi / d x \sim \sum_{j=m}^{+\infty} b_{j}(\mathbf{w}) r^{j}$ and $\phi$ has the asymptotic expansion $\phi \sim \sum_{j=m+1}^{+\infty} a_{j}(\mathbf{w}) r^{j}$. Then by the definition given in example 7

$$
\begin{aligned}
-\left\langle\operatorname{Pf}(H(x)), \frac{d \phi}{d x}\right\rangle & =-\int_{|x-a| \geq A} H(x) \frac{d \phi}{d x} d x+\int_{|x-a|<A} H(x)\left(\frac{d(\phi(x))}{d x}-\sum_{j \leq-1} b_{j}(w) r^{j}\right) d x \\
& +\sum_{j<-1} b_{j}(\mathbf{1}) \frac{A^{j+1}}{j+1}+b_{-1}(\mathbf{1}) \log A \\
& =-\int_{A}^{+\infty} \frac{d \phi}{d x} d x-\int_{0}^{A}\left(\frac{d(\phi(x))}{d x}-\sum_{j \leq-1} b_{j}(w) r^{j}\right) d x \\
& -\sum_{j<-1} b_{j}(\mathbf{1}) \frac{A^{j+1}}{j+1}-b_{-1}(\mathbf{1}) \log A \\
& =\phi(A)-\phi(A)+a_{0}(\mathbf{1})+0=a_{0}(\mathbf{1})=\left\langle g_{1} \delta_{*}, \phi\right\rangle_{\mathcal{D}_{*}^{\prime}(\mathbb{R}) \times \mathcal{D}_{*}(\mathbb{R})}
\end{aligned}
$$

Thus the derivative of the Heaviside function is $g_{1} \delta_{*}$, which is defined in the example 8

Now consider the projection of the derivative of the Heaviside function $d^{*}(P f(H(x))) / d x=$ $g_{1} \delta_{*}$ onto the usual distribution space $\mathcal{D}^{\prime}(\mathbb{R})$ :

$$
\left\langle\pi\left(g_{1} \delta_{*}\right), \phi\right\rangle=\left\langle g_{1} \delta_{*}, i(\phi)\right\rangle=\phi(0)=\langle\delta, \phi\rangle .
$$

Keep in mind that $\pi(P f(H(x)))=H(x)$, the usual Heaviside function. Hence $\pi\left(d^{*}(P f(H(x))) / d x\right)=\delta(x)=\bar{d}(\pi(P f(H(x)))) / d x$ as expected.

Next let me present an application of the derivative of thick distributions.

Problem 1. Paskusz [20] pointed out that the following proof is problematic, where $H(x)$ is the usual Heaviside function:

Since $H(x)=H^{2}(x)$, taking the distributional derivative on both sides, we have $\delta(x)=2 H(x) \delta(x)$. Hence $H(x) \delta(x)=\frac{1}{2} \delta(x)$. However, if we multiply $H(x)$ on both sides again we will get $\frac{1}{2} \delta(x)=H(x) \delta(x)=H^{2}(x) \delta(x)=\frac{1}{2} H(x) \delta(x)=$ $\frac{1}{4} \delta(x)$, hence we have $\frac{1}{2}=\frac{1}{4}$, which is clearly wrong.

The key observation of this mistake is that $H(x) \cdot H(x)$ is not a well-defined distribution, that is, $\left\langle H^{2}(x), \phi(x)\right\rangle_{\mathcal{D}^{\prime}(\mathbb{R}) \times \mathcal{D}(\mathbb{R})}=\langle H(x), H(x) \phi(x)\rangle$ is not welldefined since $H(x) \phi(x)$ is not a usual test function in $\mathcal{D}(\mathbb{R})$ : it has a jump discontinuity. Thus we cannot simply apply the distributional derivative on both sides of the equaion $H(x)=H^{2}(x)$. 
Solution 1. In the sense of thick distributions we can restate this whole story in a rigorous way thus to avoid the mistake $\frac{1}{2}=\frac{1}{4}$. In fact, it's easy to see that $H(x)$ is a multiplier of the thick distributions, i.e. $H(x) f(x) \in \mathcal{D}_{*}^{\prime}(\mathbb{R})$ for any $f(x) \in \mathcal{D}_{*}^{\prime}(\mathbb{R})$. Thus $H(x) \cdot H(x)$ should be viewed as a multiplier times a thick distribution: $H(x) \cdot \operatorname{Pf}(H(x))$. Then

$$
\begin{aligned}
\frac{d^{*}(H(x) \cdot P f(H(x)))}{d x} & =\frac{d(H(x))}{d x} \operatorname{Pf}(H(x))+H(x) \frac{d^{*}(P f(H(x)))}{d x} \\
& =0+g_{1} \delta_{*}
\end{aligned}
$$

from the previous discussions. And equation 3.22 tells us that $\pi\left(\frac{d^{*}(H(x) \cdot P f(H(x)))}{d x}\right)=$ $\delta$.

On the other hand, it is clear that $H(x) \cdot \operatorname{Pf}(H(x))=\operatorname{Pf}(H(x))$, taking derivatives on both sides yields

$$
g_{1} \delta_{*}=g_{1} \delta_{*} .
$$

\section{REFERENCES}

[1] Luc Blanchet and Guillaume Faye. Hadamard regularization. J. Math. Phys., 41(11):7675$7714,2000$.

[2] Luc Blanchet, Guillaume Faye, and Samaya Nissanke. Structure of the post-Newtonian expansion in general relativity. Phys. Rev. D (3), 72(4):044024, 10, 2005.

[3] S. M. Blinder. Delta functions in spherical coordinates and how to avoid losing them: Fields of point charges and dipoles. American Journal of Physics, 71:816-818, August 2003.

[4] J. M. Bowen. Delta function terms arising from classical point-source fields. American Journal of Physics, 62:511-515, June 1994.

[5] R. Estrada and R. P. Kanwal. Distributional analysis for discontinuous fields. J. Math. Anal. Appl., 105(2):478-490, 1985.

[6] Ricardo Estrada. The nonexistence of regularization operators. J. Math. Anal. Appl., 286(1):1-10, 2003.

[7] Ricardo Estrada and Stephen A. Fulling. Functions and distributions in spaces with thick points. Int. J. Appl. Math. Stat., 10(S07):25-37, 2007.

[8] Ricardo Estrada and Ram P. Kanwal. A distributional approach to asymptotics. Birkhäuser Advanced Texts: Basler Lehrbücher. [Birkhäuser Advanced Texts: Basel Textbooks]. Birkhäuser Boston Inc., Boston, MA, second edition, 2002. Theory and applications.

[9] Ricardo Estrada and Yunyun Yang. Applications of the thick distributional calculus. Novi Sad J. Math., 44(2):121-135, 2014.

[10] F. Farassat. Introduction to generalized functions with applications in aerodynamics and aeroacoustics. Technical report, May 1994.

[11] C. P. Frahm. Some novel delta-function identities. American Journal of Physics, 51:826-829, September 1983.

[12] J. Franklin. Comment on "Some novel delta-function identities" by Charles P. Frahm [Am. J. Phys. 51, 826-829 (1983)]. American Journal of Physics, 78:1225-1226, November 2010.

[13] Andre Gsponer. Distributions in spherical coordinates with applications to classical electrodynamics. European J. Phys., 28(2):267-275, 2007.

[14] V. Hnizdo. Generalized second-order partial derivatives of 1/r. European Journal of Physics, 32:287-297, March 2011.

[15] John Horvth. Topological Vector Spaces and Distributions I. 2012.

[16] Yu Kuang Hu. Comment on "delta functions in spherical coordinates and how to avoid losing them: Fields of point charges and dipoles," by s. m. blinder. American Journal of Physics, 72(3):409-410, 2004.

[17] H. S. Kaltenborn. Linear functional operations on functions having discontinuities of the first kind. Bull. Amer. Math. Soc., 40(10):702-708, 1934.

[18] Ram P. Kanwal. Generalized functions. Birkhäuser Boston Inc., Boston, MA, third edition, 2004. Theory and applications. 
[19] S. Lojasiewicz. Sur la valeur et la limite d'une distribution en un point. Studia Math., 16:1-36, 1957.

[20] G. F. Paskusz. Comments on "Transient analysis of energy equation of dynamical systems". IEEE Transactions on Education, 43:242, May 2000.

[21] A. Sellier. Asymptotic expansion of a class of multi-dimensional integrals. Canad. J. Math., 48(5):1079-1090, 1996.

[22] A. Sellier. Hadamard's finite part concept in dimension $n \geq 2$; definition and change of variables, associated Fubini's theorem, derivation. Math. Proc. Cambridge Philos. Soc., 122(1):131-148, 1997.

[23] François Trèves. Topological vector spaces, distributions and kernels. Academic Press, New York-London, 1967.

[24] C. Vibet. Transient analysis of energy equation of dynamical systems. IEEE Transactions on Education, 42:217-219, 1999.

[25] Y. Yang and R. Estrada. Extension of Frahm formulas for $\partial i \partial j$. Indian J. Math., 55(2):237245, 2013.

[26] Y. Yang and R. Estrada. The dual of the space of regulated functions in several variables. Sarajevo J. Math, (22):1-20, 2013.

[27] Yunyun Yang and Ricardo Estrada. Distributions in spaces with thick points. J. Math. Anal. Appl., 401(2):821-835, 2013.

[28] Yunyun Yang and Ricardo Estrada. Regularization using different surfaces and the secondorder derivatives of 1/r. Appl. Anal., 92(2):246-258, 2013.

[29] Yunyun Yang and Ricardo Estrada. Asymptotic expansion of thick distributions. Asymptot. Anal., 95(1-2):1-19, 2015.

Department of Mathematics, Hefei University of Technology

E-mail address: yangyunyun@hfut.edu.cn

URL: http://maths.hfut.edu.cn/2016/1126/c7700a213853/page.htm 